\title{
Commentary on: "Number-space mapping in the newborn chick resembles humans' mental number line"
}

\author{
Caroline B. Drucker ${ }^{1,2 *}$ and Elizabeth M. Brannon ${ }^{1,3}$ \\ ${ }^{1}$ Center for Cognitive Neuroscience, Duke University, Durham, NC, USA, ${ }^{2}$ Department of Neurobiology, Duke University, \\ Durham, NC, USA, ${ }^{3}$ Department of Psychology and Neuroscience, Duke University, Durham, NC, USA
}

Keywords: numerical cognition, spatial cognition, mental number line, birds, culture

\section{A commentary on}

Number-space mapping in the newborn chick resembles humans' mental number line by Rugani, R., Vallortigara, G., Priftis, K., and Regolin, L. (2015). Science 347 (6221), 534-536. doi: $10.1126 /$ science.aaa1379

\section{OPEN ACCESS}

Edited by:

Michael Beran,

Georgia State University, USA

Reviewed by:

Regina Paxton Gazes,

Bucknell University, USA

*Correspondence:

Caroline B. Drucker

caroline.drucker@duke.edu

Specialty section:

This article was submitted to Comparative Psychology, a section of the journal Frontiers in Psychology

Received: 04 March 2015 Accepted: 12 March 2015 Published: 27 March 2015

Citation: Drucker CB and Brannon EM (2015) Commentary on: "Number-space mapping in the newborn chick resembles humans' mental number

line". Front. Psychol. 6:352.

doi: 10.3389/fpsyg.2015.00352
When you think about the numbers one through twenty, what do you see? Most people visualize numbers laid out in space from left to right. In other words, we have a "mental number line" (Restle, 1970; Seron et al., 1992). People are actually faster at making judgments about small numbers when they are presented on the left and large numbers on the right, providing strong evidence that number is mentally mapped onto space in adults (Dehaene et al., 1993). This oft-replicated psychophysical phenomenon (Fias and Fischer, 2005) is reduced or even reversed in cultures that read from right to left, suggesting that the mental number line is a byproduct of culturally specific experiences (Zebian, 2005; Shaki and Fischer, 2008; Shaki et al., 2009). An alternative possibility is that even though the mental number line can be influenced by cultural practices it has a more fundamental place in the mind.

Recent evidence that preverbal infants (de Hevia and Spelke, 2010), birds (Rugani et al., 2007, 2010, 2011), chimpanzees (Adachi, 2014), and monkeys (Drucker and Brannon, 2014) map number onto space endorses a biological basis for the mental number line. However, these developmental and comparative studies omit a key feature of the mental number line in humans: a given number is "large" in some contexts and "small" in others. In an elegant new experimental study, Rugani and colleagues demonstrate that newborn domestic chicks (Gallus gallus) also show context-dependent spatial-numerical mapping (Rugani et al., 2015).

Rugani and colleagues first trained 3-day-old chicks to walk around a panel for a reward (Figure 1). To establish a numerical context, the panel always displayed the same number of items for a given chick: either five or twenty. Later that day, chicks were placed in an arena with two panels in front of them to the left and right displaying the same number of items. If the chick had been trained with five items, both panels either had two or eight items; if it had been trained with twenty items, the panels both showed eight or thirty-two items. A reward was placed behind each of the two identical panels. If chicks did not mentally map number onto space they should have had no preference. Astonishingly, when the number displayed on the panels was lower than the training number, chicks tended to walk to the left panel; when it was higher than the training number, they walked to the right. Crucially, the side they preferred when the test number was eight depended on training context: chicks trained with five walked to the right, whereas chicks trained with twenty walked to the left. 


\section{Training: 20 items}

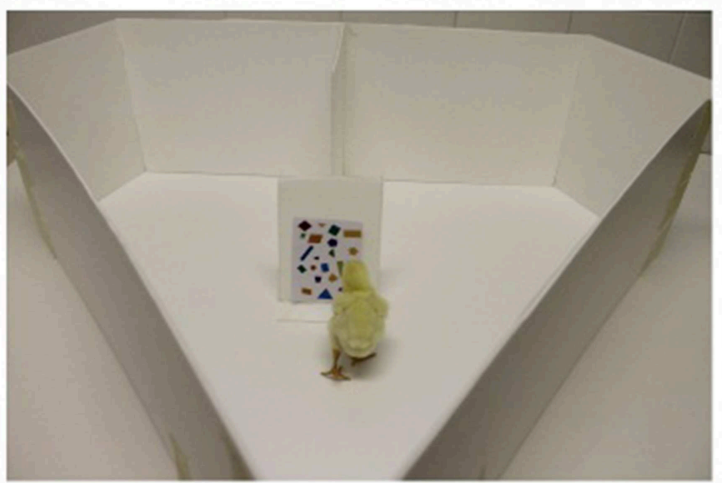

Testing: 8 items

Testing: 32 items
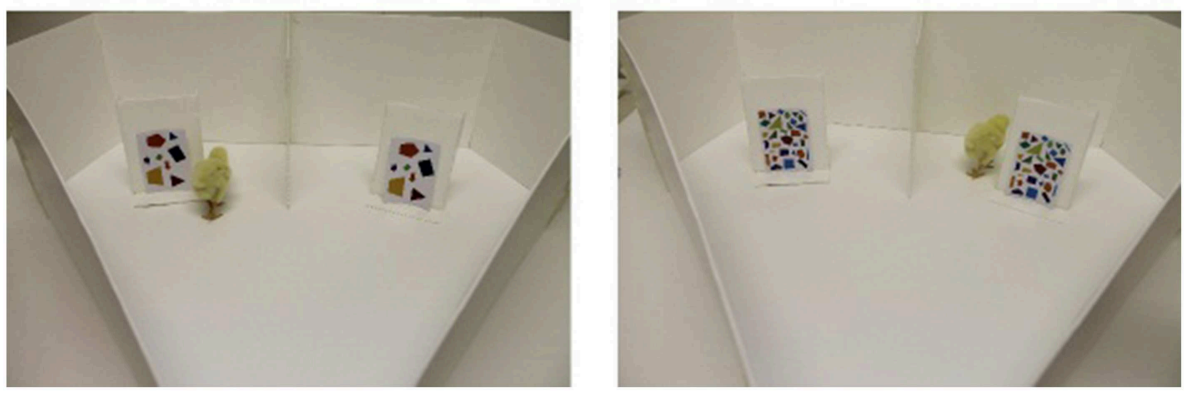

FIGURE 1 | A chick performing the task developed by Rugani et al (2015). Training with a single 20 -item panel is shown at the top. The two different testing conditions are shown at the bottom, with the low-number test (8 vs. 8) shown at left and the high-number test (32 vs. 32) shown at right. The images depict a control condition where shape, size, and color of the items were varied. Photographs courtesy of Rosa Rugani.
Control experiments ruled out other non-numerical features of the visual displays that might have influenced the chicks' choices. First Rugani and colleagues randomized the shape, color, and size of the items on the panels. Next they equated the total surface area as well as the overall spatial extent occupied by the items on each training and testing panel. Finally they equated the total perimeter and density of the items on each training and testing panel. Through all of these conditions, chicks maintained their preference for the left (or right) panel when test numbers were smaller (or larger, respectively) than the trained value. These results suggest that chicks map number-rather than some other continuous parameter that co-varies with numberonto space. In adult humans, a host of continua are mapped onto space, including temporal duration (Vallesi et al., 2008), volume of liquid (Kirjakovski and Utsuki, 2012), and even degree of emotional expression (Holmes and Lourenco, 2011). Such findings suggest a more generalized magnitude-space mapping, or a mental magnitude line. Future studies should explore whether other animals map any type of magnitude besides number to space.

Rugani and colleagues' findings provide clear evidence that chicks map number to space, and that as in humans, this mapping is context-dependent. Moreover, the 3-day-old chicks had little experience with numerical stimuli, had no exposure to cultural artifacts like keyboards portraying numbers in a line, and definitely did not read written text-largely ruling out the possi- bility that number-space mappings are learned. Rather, the tendency to map number to space seems to be an intrinsic part of representing number or more generally magnitude. Why might animals map number or magnitudes onto space?

The explanation provided by Rugani and colleagues involves hemispheric asymmetries in bird brain function. They propose that right hemisphere dominance for numerical processing produces increased right hemisphere activation-and thus increased leftward attention-when considering numerical information, which could cause birds to begin enumerating from the left (see also Vallortigara, 2012). Another possibility is that numerical representations are spatially organized in the brain. Recent functional neuroimaging evidence suggests that there is a topographical arrangement of numerical magnitudes in human parietal cortex (Harvey et al., 2013). However, this cortical map has only been found in humans, and only for the numbers one to seven.

Another idea is that numerical and spatial cognition rely on common neural circuits (Hubbard et al., 2005). The same regions of parietal cortex-in particular, the lateral and ventral intraparietal areas-play a role in processing both number and spatial attention in primates. Activation of these regions by numerical information could therefore lead to shifts in spatial attention. This hypothesis is attractive from an evolutionary perspective, since navigating space is a fundamental problem faced by all animals. Rather than developing new cognitive systems to deal with 
abstract concepts of "less" and "more," it would be more efficient to re-use the system already in place for processing space (Holmes and Lourenco, 2011). Considering numerical processing as an exaptation (Gould and Vrba, 1982) of spatial processing could explain the functional link between these two systems.

An important caveat to these hypotheses is that one-to-one homologies between avian and mammalian brain regions remain elusive (Jarvis et al., 2005). In particular, number representation has not been localized in the avian brain. Exploring where and

\section{References}

Adachi, I. (2014). Spontaneous spatial mapping of learned sequence in chimpanzees: evidence for a SNARC-like effect. PLoS ONE 9:e90373. doi: 10.1371/journal.pone.0090373

de Hevia, M. D., and Spelke, E. S. (2010). Number-space mapping in human infants. Psychol. Sci. 21, 653-660. doi: 10.1177/0956797610366091

Dehaene, S., Bossini, S., and Giraux, P. (1993). The mental representation of parity and number magnitude. J. Exp. Psychol. Gen. 122, 371. doi: 10.1037/00963445.122.3.371

Drucker, C. B., and Brannon, E. M. (2014). Rhesus monkeys (Macaca mulatta) map number onto space. Cognition 132, 57-67. doi: 10.1016/j.cognition.2014.03.011

Fias, W., and Fischer, M. H. (2005). "Spatial representation of number," in Handbook of Mathematical Cognition, ed J. Campbell (New York, NY: Pschology Press), 43-54.

Gould, S. J., and Vrba, E. S. (1982). Exaptation - a missing term in the science of form. Paleobiology 8, 4-15.

Harvey, B. M., Klein, B. P., Petridou, N., and Dumoulin, S. O. (2013). Topographic representation of numerosity in the human parietal cortex. Science 341, 1123-1126. doi: 10.1126/science.1239052

Holmes, K. J., and Lourenco, S. F. (2011). Common spatial organization of number and emotional expression: a mental magnitude line. Brain Cogn. 77, 315-323. doi: 10.1016/j.bandc.2011.07.002

Hubbard, E. M., Piazza, M., Pinel, P., and Dehaene, S. (2005). Interactions between number and space in parietal cortex. Nat. Rev. Neurosci. 6, 435-448. doi: $10.1038 / \mathrm{nrn} 1684$

Jarvis, E. D., Güntürkün, O., Bruce, L., Csillag, A., Karten, H., Kuenzel, W., et al. (2005). Avian brains and a new understanding of vertebrate brain evolution. Nat. Rev. Neurosci. 6, 151-159. doi: 10.1038/nrn1606

Kirjakovski, A., and Utsuki, N. (2012). From SNARC to SQUARC: universal mental quantity line? Int. J. Psychol. Stud. 4, 217-227. doi: 10.5539/ijps. $\mathrm{v} 4 \mathrm{n} 2 \mathrm{p} 217$

Restle, F. (1970). Speed of adding and comparing numbers. J. Exp. Psychol. 83, 274.

Rugani, R., Kelly, D., and Szelest, I. (2010). Is it only humans that count from left to right? Biol. Lett. 6, 290-292. doi: 10.1098/rsbl.2009.0960

Rugani, R., Regolin, L., and Vallortigara, G. (2007). Rudimental numerical competence in 5-day-old domestic chicks (Gallus gallus): identification of ordinal how bird brains process numbers could shed light on the origins of the mental number line.

\section{Acknowledgments}

We would like to thank Dr. Michael Platt for helpful discussion of this manuscript. This material is based upon work supported by the National Science Foundation Graduate Research Fellowship Program under Grant No. 1106401 to CBD.

position. J. Exp. Psychol. Anim. Behav. Process. 33, 21-31. doi: 10.1037/00977403.33.1.21

Rugani, R., Vallortigara, G., Priftis, K., and Regolin, L. (2015). Number-space mapping in the newborn chick resembles humans' mental number line. Science 347, 534-536. doi: 10.1126/science.aaa1379

Rugani, R., Vallortigara, G., Vallini, B., and Regolin, L. (2011). Asymmetrical number-space mapping in the avian brain. Neurobiol. Learn. Mem. 95, 231-238. doi: 10.1016/j.nlm.2010.11.012

Seron, X., Pesenti, M., Noël, M.-P., Deloche, G., and Cornet, J.-A. (1992). Images of numbers, or "When 98 is upper left and 6 sky blue." Cognition 44, 159-196.

Shaki, S., and Fischer, M. (2008). Reading space into numbers-a crosslinguistic comparison of the SNARC effect. Cognition 108, 590-599. doi: 10.1016/j.cognition.2008.04.001

Shaki, S., Fischer, M. H., and Petrusic, W. M. (2009). Reading habits for both words and numbers contribute to the SNARC effect. Psychon. Bull. Rev. 16, 328-331. doi: $10.3758 /$ PBR.16.2.328

Vallesi, A., Binns, M. A., and Shallice, T. (2008). An effect of spatial-temporal association of response codes: understanding the cognitive representations of time. Cognition 107, 501-527. doi: 10.1016/j.cognition.2007.10.011

Vallortigara, G. (2012). Core knowledge of object, number, and geometry: a comparative and neural approach. Cogn. Neuropsychol. 29, 213-236. doi: $10.1080 / 02643294.2012 .654772$

Zebian, S. (2005). Linkages between number concepts, spatial thinking, and directionality of writing: the SNARC effect and the reverse SNARC effect in English and Arabic monoliterates, biliterates, and illiterate Arabic speakers. J. Cogn. Cult. 5, 165-190. doi: 10.1163/1568537054068660

Conflict of Interest Statement: The authors declare that the research was conducted in the absence of any commercial or financial relationships that could be construed as a potential conflict of interest.

Copyright (C) 2015 Drucker and Brannon. This is an open-access article distributed under the terms of the Creative Commons Attribution License (CC BY). The use, distribution or reproduction in other forums is permitted, provided the original author(s) or licensor are credited and that the original publication in this journal is cited, in accordance with accepted academic practice. No use, distribution or reproduction is permitted which does not comply with these terms. 